\title{
Spermine-NBD as fluorescent probe for studies of the polyamine transport system in Leishmania donovani
}

Elodie Jagu ${ }^{\mathrm{a}}$, Sébastien Pomel ${ }^{\mathrm{b}}$, Stéphanie Pethe ${ }^{\mathrm{a}}$, Jean-Christophe Cintrat, ${ }^{\mathrm{c}}$ Philippe M. Loiseau ${ }^{\mathrm{b}}$, Raphaël Labruère ${ }^{\mathrm{a} *}$

a- Institut de Chimie Moléculaire et des Matériaux d'Orsay (ICMMO), CNRS, Univ Paris Sud, Université Paris-Saclay, 15 rue Georges Clemenceau, 91405 Orsay Cedex, France

b- Chimiothérapie antiparasitaire, UMR 8076 BioCis, CNRS, Univ Paris Sud, Université ParisSaclay, 5 rue Jean-Baptiste Clément, 92290 Châtenay-Malabry, France

c- Service de Chimie Bio-organique et Marquage (SCBM), CEA, Université Paris-Saclay, 91191 Gif/Yvette, France

Keywords: Leishmania donovani, Polyamine transport, Radioisotopic method, fluorescence

* Corresponding author

E-mail address: raphael.labruere@u-psud.fr (R. Labruère).

\begin{abstract}
This study describes the synthesis of fluorescent probes as potential substrates for the polyamine transport system (PTS) of Leishmania donovani. A competitive radioassay was used to determine the most efficient probe. We observed that the conjugate sperminenitrobenzofurazan (Spm-NBD) was able to compete with $\left[{ }^{3} \mathrm{H}\right]$-spermidine in L. donovani at a potent $\mathrm{IC}_{50}$ of $60 \mu \mathrm{M}$.
\end{abstract}


During their life cycle, Kinetoplastids have to face different biological environments within their insect or mammalian host. These environments can exhibit a vastly different composition of nutrients, and parasites need a strong capacity to acclimatize for good metabolic activity. Kinetoplastids require these nutrients for survival and growth. The replacement of biosynthetic pathways by transport systems is favorable since the energy required to import a nutrient is typically lower than its biosynthesis. Polyamines are essential compounds for parasites (Figure 1) and membrane transporters can uptake polyamines from the extracellular environment. ${ }^{1}$ These uptake systems are well described in bacteria and yeast but are poorly characterized at the molecular level for mammalian and protozoan parasites. However, the biochemical functionality of transporters has been studied in some protozoa. ${ }^{2-6}$

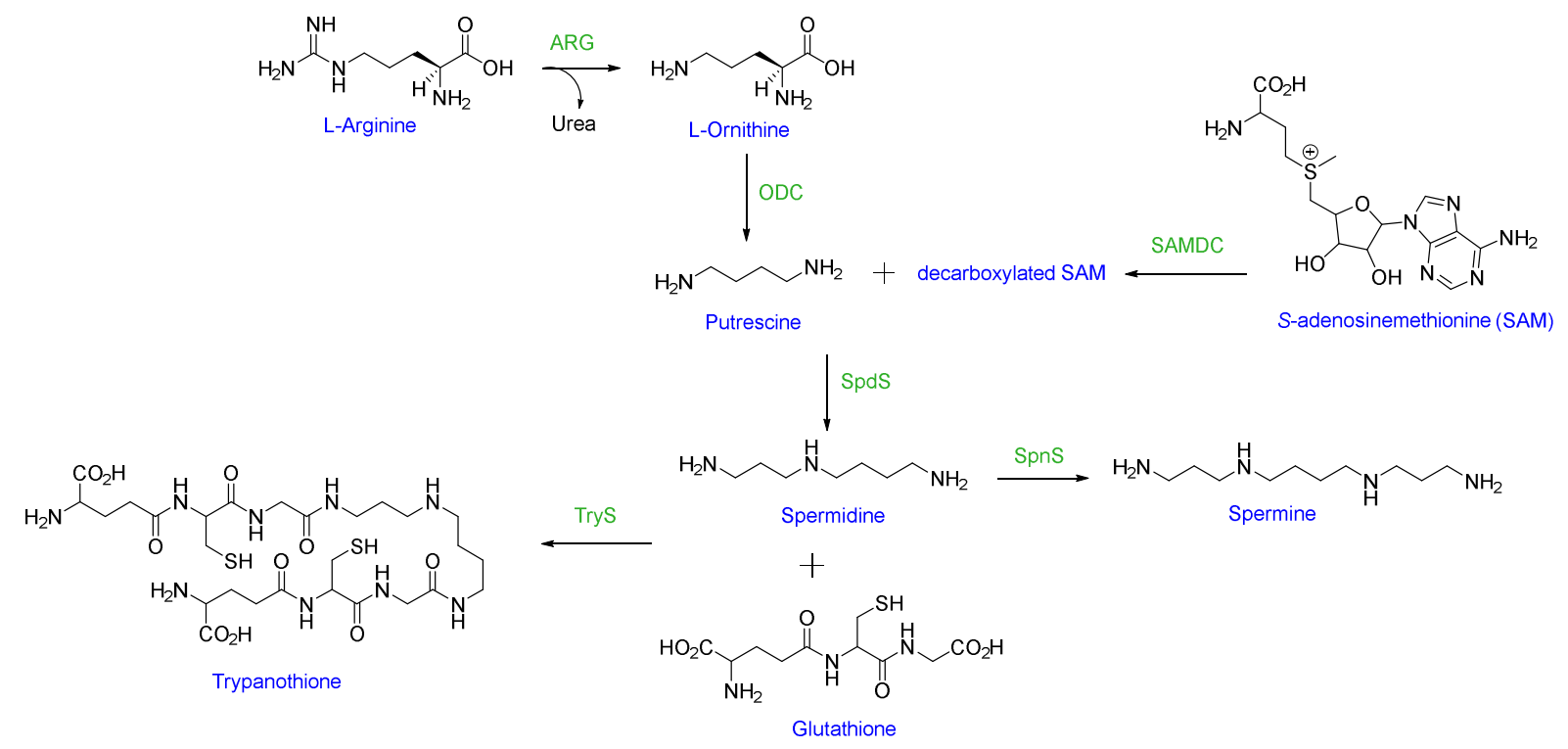

Figure 1: Trypanothione and polyamine biosynthetic pathway. ARG: arginase, ODC: ornithine decarboxylase, SAMDC: Sadenosinemethione decarboxylase, SpdS spermidine synthetase, SpnS: spermine synthetase, TryS: trypanothione synthetase.

Leishmania infantum was the first Leishmania species in which a polyamine transport system has been described. ${ }^{7}$ This transport system for putrescine was identified and characterized by measuring the uptake of radioactively labelled putrescine into the promastigote form of the parasite. These results have been confirmed in Leishmania donovani promastigotes in which a second polyamine transporter has been described for spermidine. ${ }^{8}$ The influx of spermidine was seven times more effective than putrescine but the affinity of both spermidine and putrescine transporters was the same for the respective polyamines. The 
transport of putrescine and spermidine has also been described in Leishmania mexicana promastigote and amastigote forms. ${ }^{9}$ In the latter study, the transport was found to be temperature- and $\mathrm{pH}$-dependent. Results from kinetic analyses confirm that putrescine and spermidine use different transporters. Hence, total understanding and molecular characterization of polyamine transporters in Kinetoplastids is far from being achieved.

In addition, polyamine transport systems can be highjacked to deliver drugs. ${ }^{10-13}$ However, it is important to prove that polyamine-analogues are incorporated via the polyamine transport system. The most widely used method for measuring polyamine transport is the radioisotopic method that measures the radioactivity uptake with radiolabeled polyamines. Despite the fact that this method is very accurate and explicit, it is expensive and needs an appropriate laboratory with authorized workers. Therefore, we became interested in identifying a fluorescent probe able to selectively compete with natural polyamines, which would allow the accurate determination of the affinity of structurally diverse polyamines for the polyamine transport system. To our knowledge, such probe has not been reported so far in Kinetoplastids. Several fluorescent polyamine probes were previously synthesized in order to study polyamine transporters in mammalian cells. Cullis et al. ${ }^{14}$ prepared polyamines conjugated with the fluorescent chromophore MANT ( $N$-methylanthraniloyl), 1 and 2, in order to localize the modified polyamines (Figure 2). Flow cytometry studies have shown that spermidine-MANT derivatives were taken up rapidly in Chinese hamster ovary cell line $(\mathrm{CHO})$ with a plateau within several hours. With a PTS-deficient mutant (CHO-MG), the authors demonstrated that non-specific uptake was very slow. Moreover, observations using conventional fluorescence microscopy provided clear evidence that the polyaminefluorophore was internalized. Furthermore, a fluorescence-based assay was published identifying the activity of the polyamine transport system in tumor cells. ${ }^{15}$ The authors synthesized a series of spermine conjugates to different fluorophores: phenylxanthenes, rhodamine, lissamine, benzofuranes such as 4-chloro-5-benzofurazan (NBD) and bodipy. Potential fluorescent probes were chosen on the basis of their optimal excitation/emission wavelength, quantum yield, selectivity for PTS-positive cells and resistance to bleaching upon light irradiation. All the synthesized fluorescent probes were evaluated for spectral properties, and their cellular incorporation was also tested in competition assays against the drug F14512, a spermine-epipodophyllotoxin conjugate. ${ }^{16}$ In addition, probe internalization 
was compared between $\mathrm{CHO}$ cells and its PTS-deficient mutant (CHO-MG) in order to attest specific incorporation of fluorescent probes by a polyamine transporter. The best fluorescent polyamine probes identified were $N^{1}$-spermine-nitrobenzoxadiazole 3 and $N^{1}$ methylspermine-nitrobenzoxadiazole 4 (Figure 2) which displayed high fluorescence intensity, strong competition with the drug F14512, and marked differential uptake in $\mathrm{CHO}$ and CHO-MG cells.

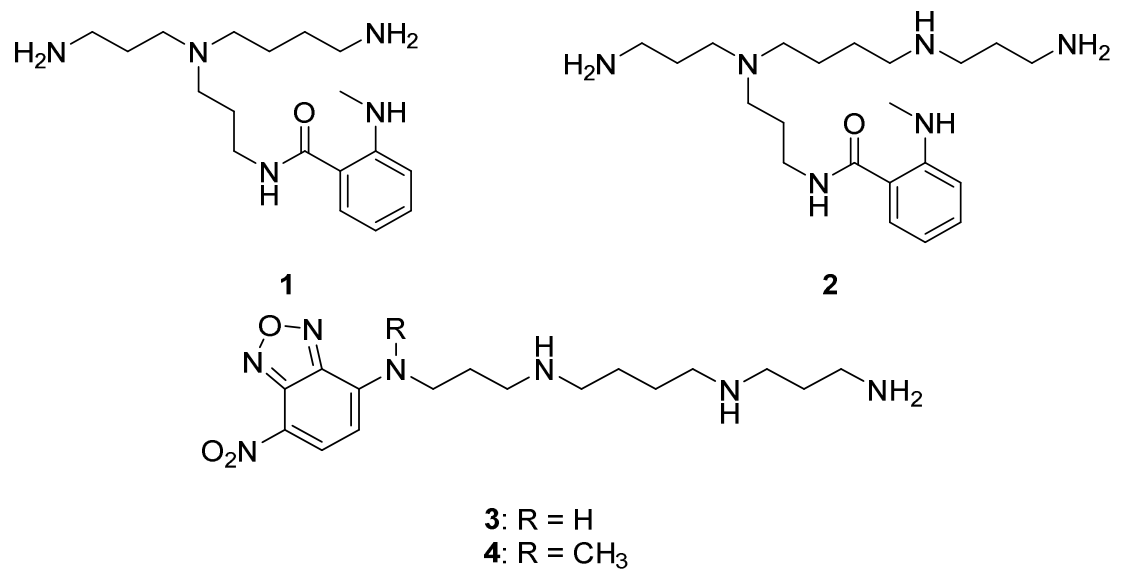

Figure 2: MANT and NBD polyamine probes..$^{14,15}$

Based on the latter study, we hypothesized that the reported "polyamine-fluorophore" conjugates could be also efficient in Trypanosomatids. We directly used the most relevant probes from the work published earlier, ${ }^{15}$ examined the behavior of several polyamine fluorescent probes with Leishmania donovani and determined whether incorporation into parasites was achieved through a polyamine transport system.

Five probes were envisioned for the study of the Kinetoplastid polyamine transport system. Putrescine and spermine derivatives had already been synthesized by Guminski et al. ${ }^{15}$ In addition, we chose to prepare two spermidine probes attached to the two different terminal amines. Each probe was obtained from the nucleophilic aromatic substitution of 7-chloro-4nitrobenzofurazan (NBD-chloride) (Scheme 1). Fluorescent control 6 displaying no polyamine moiety was obtained by reacting methylamine and NBD-chloride in methanol in $61 \%$ yield. All the polyamine probes were prepared according to protocols described by Guminski et al. ${ }^{15}$ Mono-protected putrescine 5a was reacted with NBD-chloride to give an intermediate Boc-putrescine-NBD $7 a$ in $50 \%$ yield after recrystallization from diisopropyl ether. Boc- 
protected spermidine/spermine (5b-d) and NBD-chloride were coupled according to a general procedure in which the primary amine was reacted with NBD-chloride in presence of $\mathrm{Cs}_{2} \mathrm{CO}_{3}$ in acetonitrile to give protected intermediates $\mathbf{7 b}, \mathbf{7 c}$ and $\mathbf{7 d}$ in yields ranging from 21 to $80 \%$ after purification on flash column chromatography. All these intermediates were deprotected using the same conditions, i.e. stirring overnight in an $\mathrm{HCl}$ solution $4 \mathrm{M}$ in dioxane to afford 8a (put-NBD) as a putrescine derivative, $\mathbf{8 d}$ (Spm-NBD) as a spermine derivative, and $\mathbf{8 c}\left(\boldsymbol{N}^{1}-\mathbf{S p d}-\mathbf{N B D}\right)$ and $\mathbf{8 b}\left(\boldsymbol{N}^{3}-\mathbf{S p d}-\mathbf{N B D}\right)$ as spermidine derivatives coupled with different nitrogen atoms of this unsymmetrical polyamine. From these two steps, the global yields were good, except for $\mathbf{8 d}(21 \%)$ and $\mathbf{8 a}(25 \%)$. All the compounds were used directly without any synthesis optimization.
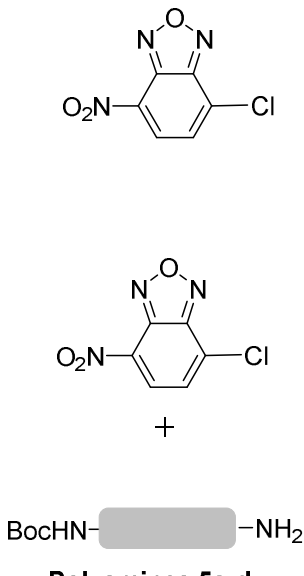

Polyamines 5a-d

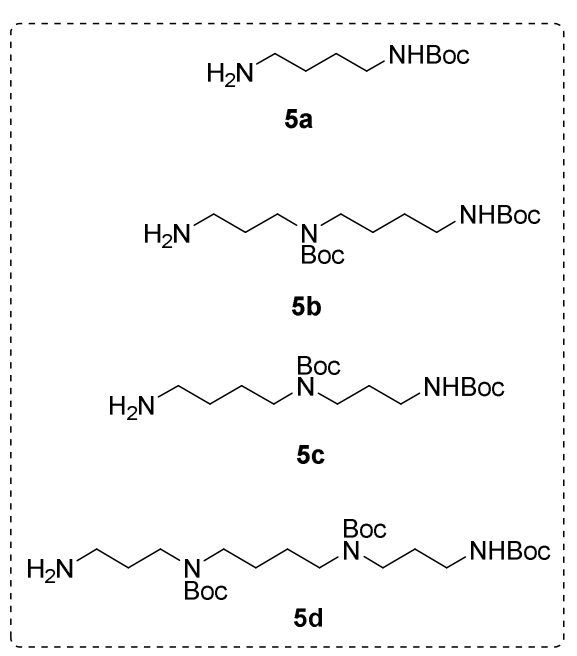

a, b

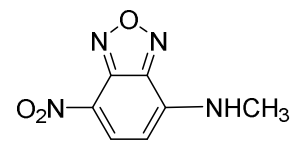

6 
In order to ensure that the fluorescent probes use a polyamine transporter, we used a radiolabeled competitive assay with $\left[{ }^{3} \mathrm{H}\right]$-spermidine. Spermidine transport in Leishmania donovani promastigotes was studied with $\left[{ }^{3} \mathrm{H}\right]$-tetrahydrochloride spermidine and competition assays were carried out with three NBD-probes (spermidine and spermine) and the control methyl-NBD 6.

First of all, an optimization was carried out to find a suitable concentration of radiolabeled ligand and the time needed for incubation. Experiments were performed at a concentration of $10 \mu \mathrm{M}$ of $\left[{ }^{3} \mathrm{H}\right]$-spermidine and with a reaction time of $10 \mathrm{~min}$. To establish the efficiency of the transporter, $\left[{ }^{3} \mathrm{H}\right]$-spermidine transport was measured in the presence of the natural substrate spermidine. In the presence of cold spermidine, transport of radiolabeled spermidine was inhibited with an $\mathrm{IC}_{50}$ value of $34 \mu \mathrm{M}$ (Figure 3). A second control consisted in evaluating the inhibition of the fluorophore not coupled to polyamine. 6 did not inhibit the $\left[{ }^{3} \mathrm{H}\right]$-spermidine transport, and was clearly not actively taken up by the spermidine transporter. This result confirms that internalization of 6 does not use a polyamine transporter.

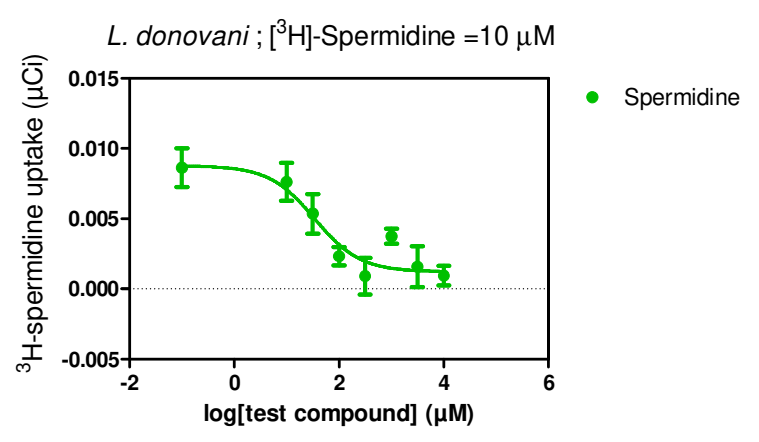

Figure 3: Competition assay with cold spermidine.

Next, the polyamine probes were evaluated using the same method. Each compound was tested in a range of concentrations from $0.1 \mu \mathrm{M}$ to $10 \mathrm{mM}$ (figure 4). $\mathbf{N}^{3}$-Spd-NBD (8b) did compete with the radiolabeled spermidine, although a relatively high concentration of the probe was needed $\left(I_{50}=475 \mu \mathrm{M}\right)$. $\mathbf{N}^{1}$-Spd-NBDb (8c) and Spm-NBD (8d) probes were however better at competing with radiolabeled spermidine. In particular, spermine 
conjugate Spm-NBD (8d) had an IC 50 of $60 \mu \mathrm{M}$ whereas $\boldsymbol{N}^{\mathbf{1}}$-Spd-NBDb (8c) had an IC 50 of 177 $\mu \mathrm{M}$.
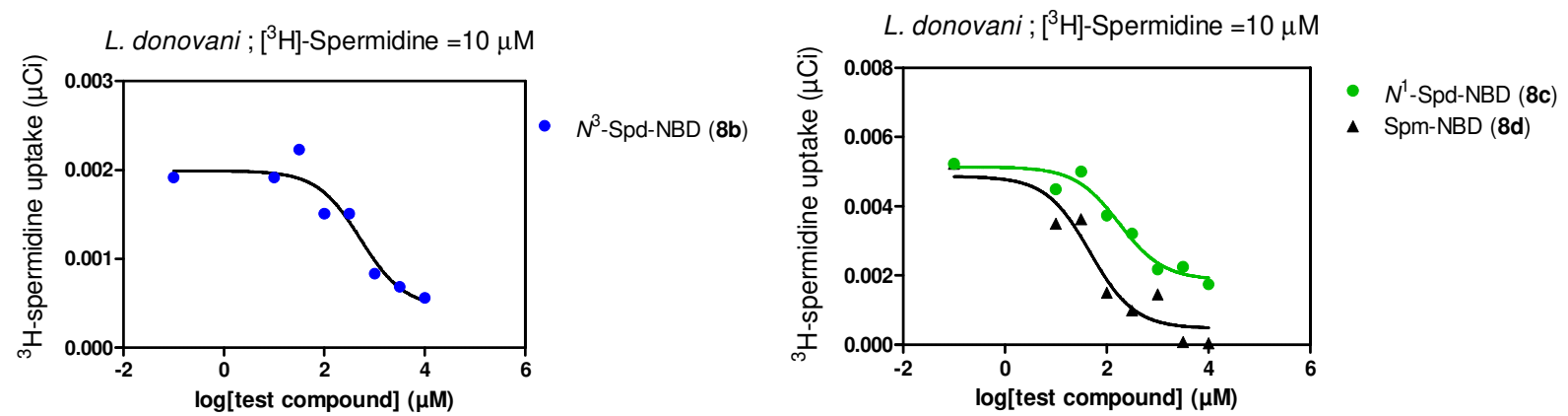

Figure 4: Competition assay with spermidine and spermine probes.

Figure 5 summarizes the inhibition capacity of the polyamine probes. The ability of the $L$. donovani promastigotes to uptake $\left[{ }^{3} \mathrm{H}\right]$-spermidine was evaluated in the presence of unlabelled spermidine and in the presence of fluorescent probes, each at concentrations of 100,300 or $1000 \mu \mathrm{M}$. Results are plotted as the percentage of spermidine uptake obtained without inhibitor (control).

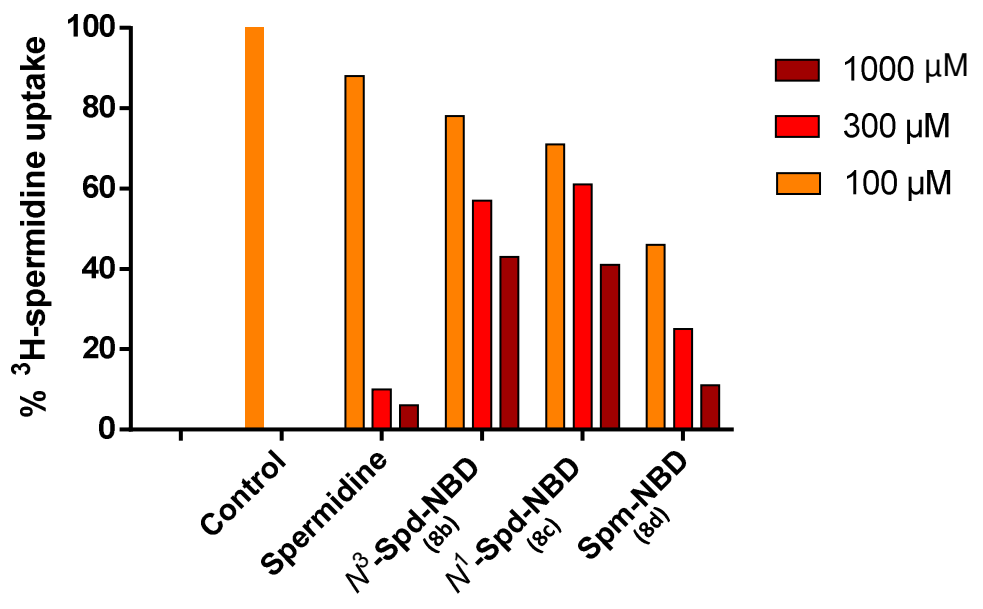

Figure 5: Competition profile of polyamine probes. Control represents the percentage of $\left[{ }^{3} \mathrm{H}\right]$-spermidine uptake in absence of inhibitor.

In conclusion, we have prepared different polyamine-nitrobenzofurazan conjugates and evaluated them as ligands of the polyamine transport system of $L$. donovani. The affinity of 
each fluorescent probe for the transporter was determined by a competition assay using the radiolabeled natural substrate $\left[{ }^{3} \mathrm{H}\right]$-spermidine. Among the different probes, the sperminenitrobenzofurazan Spm-NBD (8d) was the most active with an $\mathrm{IC}_{50}$ of $60 \mu \mathrm{M}$ emphasizing the potent internalization of this useful fluorescent polyamine by the spermidine transporter of Leishmania donovani promastigote.

\section{Acknowledgement}

This work was supported by Initiative d'Excellence Paris-Saclay (IdEx). The authors are grateful to the COST Action CM1307 and to Laura Darai for technical assistance on organic synthesis. We would also acknowledge Dr. Darren A. Smith for his valuable contribution in proofreading the manuscript.

\section{References}

[1] Ilari, A.; Fiorillo, A.; Genovese, I.; Colotti, G. Future Med. Chem. 2017, 9, 61-77.

[2] Persson, K.; Aslund, L.; Grahn, B.; Hanke, J.; Heby, O. Biochem. J. 1998, 333, 527-537.

[3] Hamana, K.; Matsuzaki, S. FEMS Microbiol. Lett. 1987, 41, 211-215.

[4] Carrillo, C.; Canepa, G. E.; Algranati, I. D.; Pereira, C. A. Biochem. Biophys. Res. Commun. 2006, 344, 936-940

[5] Ariyanayagam, M. R.; Fairlamb, A. H. Mol. Biochem. Parasitol. 1997, 84, 111-121.

[6] Reigada, C.; Sayé, M.; Vera, E. V.; Balcazar, D.; Fraccaroli, L.; Carrillo, C.; Miranda, M. R.; Pereira, C. A. J. Membr. Biol. 2016, 249, 475-481.

[7] Balaña-Fouce, R.; Ordóñez, D.; Alunda, J. M. Mol. Biochem. Parasitol. 1989, 35, 43-50.

[8] Kandpal, M.; Tekwani, B. L. Life Sci. 1997, 60, 1793-1801.

[9] Basselin, M.; Coombs, G. H.; Barrett, M. P. Mol. Biochem. Parasitol. 2000, 109, 37-46.

[10] Bacchi, C. J.; Nathan, H. C.; Hutner, S. H.; McCann, P. P.; Sjoerdsma, A. Science 1980, 210, 332-334.

[11] Jagu, E.; Pomel, S.; Pethe, S.; Loiseau, P.M.; Labruère, R. Eur. J. Med. Chem. 2017, 139, 982-1015.

[12] Mukhopadhyay, R.; Madhubala, R. Exp. Parasitol. 1995, 81, 39-46.

[13] Lizzi, F.; Veronesi, G.; Belluti, F.; Bergamini, C.; López-Sánchez, A.; Kaiser, M.; Brun, R.; Krauth-Siegel, R. L.; Hall, D. G.; Rivas, L.; Bolognesi, M. L. J. Med. Chem. 2012, 55, 1049010500.

[14] Cullis, P. M.; Green, R. E.; Merson-Davies, L.; Travis, N. Chem. Biol. 1999, 6, 717-729.

[15] Guminski, Y.; Grousseaud, M.; Cugnasse, S.; Brel, V.; Annereau, J.-P.; Vispé, S.; Guilbaud, N.; Barret, J.-M.; Bailly, C.; Imbert, T. Bioorg. Med. Chem. Lett. 2009, 19, 2474-2477.

[16] Barret, J.-M.; Kruczynski, A.; Vispé, S.; Annereau, J.-P.; Brel, V.; Guminski, Y.; Delcros, J.G.; Lansiaux, A.; Guilbaud, N.; Imbert, T.; Bailly, C. Cancer Res. 2008, 68, 9845-9853. 


\section{Leishmania donovani}
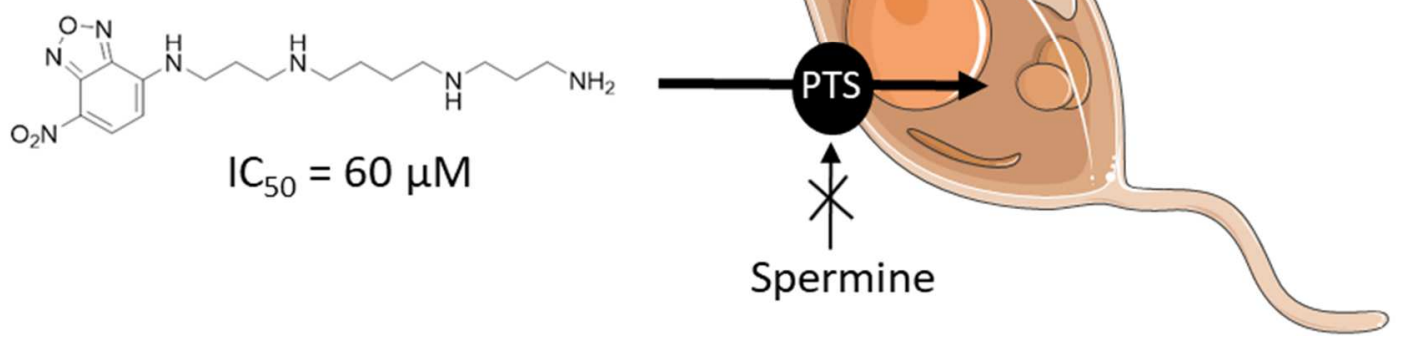